raised in patients with diabetes, and most markedly in women. Patients with type 1 diabetes had absolute risks of CVD equivalent to those of much older individuals in the general population.

The authors conclude that patients with type 1 diabetes have extremely high absolute and relative risks of CVD, despite recent advances in health care. They urge that clinicians are made aware of these increased risks so that patients who could benefit from cardiovascular treatment are not overlooked.

Original article Soedamah-Muthu SS et al. (2006) High risk of cardiovascular disease in patients with type 1 diabetes in the U.K.: a cohort study using the General Practice Research Database. Diabetes Care 29: 798-804

\section{Septal morphology is related to genotype in hypertrophic cardiomyopathy}

Given that hypertrophic cardiomyopathy (HCM) is a genetically heterogeneous condition, screening the relatives of affected patients is an important aspect of clinical care. Screening DNA samples for underlying HCM-causing mutations, however, can be expensive. As features of the ventricular septum and disease progression can vary between patients with HCM, Binder et al. have investigated whether morphological differences detected by echocardiography could be useful for selecting patients for genetic testing.

Two independent reviewers evaluated echocardiographic data from 382 unrelated patients with HCM. They graded the morphological features of the septal contour without knowledge of the results of a mutation screen of eight myofilament-encoding genes.

The presence or absence of a reverse curvature septal morphology correctly predicted a positive or negative genotype in $82 \%$ of patients $(n=315 ; 73 \%$ sensitivity and $89 \%$ specificity). Notably, older patients (aged $\geq 50$ years; $n=126$ ) were more likely to have a sigmoid septal contour than younger individuals ( $n=256 ; 67 \%$ vs $38 \% ; P<0.001)$, and only a small proportion (23\%) of the older group had identifiable mutations in a myofilamentencoding gene. By contrast, genetic screening yielded mutations in $44 \%$ of the younger patients $(P<0.001)$. The authors conclude that using echocardiography to characterize the septal contour in patients with $\mathrm{HCM}$ could facilitate strategic genotyping.

Original article Binder J et al. (2006) Echocardiographyguided genetic testing in hypertrophic cardiomyopathy: septal morphological features predict the presence of myofilament mutations. Mayo Clin Proc 81: 459-467

\section{Ethnic differences in risk of MI among patients with ESRD}

Among patients with chronic renal insufficiency, cardiovascular disease (CVD) risk is higher in racial and ethnic minorities than in whites. Young et al. have investigated whether there are ethnic differences in incident myocardial infarction (MI) rates among patients with end-stage renal disease (ESRD).

The analysis included 271,102 patients who initiated renal replacement therapy in the US between January 1995 and December 2000, and were eligible for Medicare insurance coverage. Prevalent CVD was present in $43.8 \%$ of patients. Overall, $52.1 \%$ of participants were white, $31.6 \%$ were African American, 3.0\% were Asian American and $11.5 \%$ were Hispanic.

Prevalent CVD was most common in whites (60.0\% of all CVD cases), followed by African Americans (25.6\%), Hispanics (10.1\%), Asian Americans (2.57\%) and other ethnicities (1.69\%). Unadjusted MI event rates were approximately twice as high in patients with prevalent CVD than in those without CVD. Factors associated with higher nonfatal and combined (i.e. fatal and nonfatal) $\mathrm{Ml}$ rates among patients with and without CVD were white race, increasing age, and inability to walk. Among patients with prevalent CVD, whites had the highest risk of combined $\mathrm{MI}$; risk was 35\% lower in African Americans, 26\% lower in Asian Americans, and 28\% lower in Hispanics, after adjustment for confounding factors. Whites also showed the greatest risk of combined $\mathrm{Ml}$ among patients without prevalent CVD.

The authors conclude that incident $\mathrm{MI}$ risk is higher in ESRD patients with prevalent CVD than in those without, and that MI risk among ESRD patients is higher in whites than in other ethnic groups.

Original article Young BA et al. (2006) Racial and ethnic differences in incident myocardial infarction in end-stage renal disease patients: the USRDS. Kidney Int 69: 1691-1698 